\title{
Incidental Findings on Upper Airway CT Images in Patients with Sleep-Disordered Breathing
}

\author{
Sangjun Kim, MD, Sang Hoo Park, MD, Woo Yong Bae, MD, PhD \\ Department of Otorhinolaryngology-Head and Neck surgery, Dong-A University College of Medicine, Busan, Korea
}

Received: April 9, 2020

Revised: June 10, 2020

Accepted: July 20, 2020

Correspondence

Woo Yong Bae, $\mathrm{MD}, \mathrm{PhD}$

Department of Otorhinolaryngology-

Head and Neck Surgery,

Dong-A University College of Medicine,

26 Daesingongwon-ro,

Seo-gu, Busan 49201, Korea

Tel $+82-51-240-5428$

Fax +82-51-253-0712

E-maildoncamel@dau.ac.kr

ORCID

Sangjun Kim

https://orcid.org/0000-0002-6025-6775

Sang Hoo Park

https://orcid.org/0000-0002-5994-2428

Woo Yong Bae

https://orcid.org/0000-0001-5578-0225

(c) This is an Open Access article distributed under the terms of the Creative Commons Attribution Non-Commercial License (https://creativecommons.org/licenses/by-nc/4.0) which permits unrestricted non-commercial use, distribution, and reproduction in any medium, provided the original work is properly cited.
Background and Objective To evaluate incidental findings and upper airway anatomical factors associated with sleep-related breathing disorder (SRBD) on upper airway CT (UACT).

Methods A total of 378 participants (304 males, 74 females) were evaluated using medical records, UACT, and polysomnography. UACT was performed from the skull base to the carina level of the distal trachea to examine the upper airway.

Results Of the 378 patients evaluated, 74 had lesions that were unrelated to SRBD. Among these patients (male: 59, female: 15) there were 32 cases of sinusitis, 16 cases of thyroid disease, 6 cases of lymphadenopathy, 6 cases of brain lesions, 3 cases of pulmonary tuberculosis, 2 cases of vallecular cyst, 1 case of pulmonary nodule, and 9 other cases. In this case, a total of 25 patients underwent medical treatment, and 11 had surgical treatment.

Conclusions Clinically important diseases can also be detected incidentally from UACT, and it is necessary to carefully evaluate and manage the suspected diseases.

Sleep Med Res 2020;11(2):116-120

Key Words Sleep-disordered breathing, Computed tomography, Sinusitis, Tumor.

\section{INTRODUCTION}

By way of definition, sleep-disordered breathing is defined as an obstruction of the upper airway during sleep that can result in ventilation disorders, such as apnea or hypopnea. Increasing obesity and metabolic syndrome in the population are associated with the increasing prevalence of this condition [1]. Furthermore, this condition can be diagnosed through many different methods, although polysomnography (PSG) is the gold standard for most diagnosis procedures and provides sufficient information to this end. In most cases, PSG can be used to diagnose whether patients have obstructive sleep apnea (OSA) or central sleep apnea by recording chest and abdominal wall movement with respiratory effort [2]. However, PSG cannot be used to specifically determine where the obstructive lesions are located in the patient. Therefore, there has been a great deal of research aimed towards identifying measures to determine the area related to the specifics of apnea development. Until now, methods using imaging modalities, from plain images to fluoroscopy, CT, and MRI, as well as endoscopic measures using the Mueller method, measures using reflected sound waves, and methods involving measurement of pressure, have been used in this research area [3]. Recent studies report the use of cone beam CT to reconstruct the upper airway or dynamic three-dimensional CT to produce 3D dynamic images for evaluation of the upper airway [4,5]. Upper airway CT (UACT) used in our institution provides images that encompass a wide area in addition to the upper airway because it obtains axial, coronal, and sagittal images of the entire upper airway from the base of the skull to the carina level of the distal trachea as a resource to find the obstructive lesions. This re- 
search has resulted in the discovery of incidental findings that were not anticipated as the primary objectives. Going further, this study demonstrates more information related to the incidentally found diseases that were discovered during UACT images, which were primarily conducted to determine the area involved in upper airway obstruction.

\section{METHODS}

\section{Subjects}

In general, the study population consisted of 378 patients who visited the otorhinolaryngology department of the author's institution within a 5-year period from June 2010 to June 2015, with a chief complaint of snoring or sleep apnea that was examined using both PSG and UACT. All patients diagnosed as OSA with PSG were imaged with UACT in our institution on a routine basis. Of the 378 patients, 304 were male and 74 were female, and they ranged in age from 12 years to 79 years (average: 48 years).

\section{Study Design}

As were seen, the lesions discovered by chance, other than manifestations that can account for sleep respiratory disturbance, were subjected to retrospective analysis with medical records. The institutional ethics committee approval was granted by our institution's IRB (DAUHIRB-17-113).

\section{Institution's Protocol for Diagnosing and Treating OSA Patients}

It is well-known that our institution operates a sleep clinic in which the neurology and oral and maxillofacial surgery departments collaborate with the otorhinolaryngology department to treat and diagnose sleep-disordered breathing. In this instance, PSG was conducted first to determine the cause and severity of sleep-disordered breathing. In the otorhinolaryngology department, UACT and drug-induced sleep endoscopy were performed to determine the anatomical structures responsible for sleep apnea, and operations such as septoplasty, tonsillectomy, or uvulectomy were performed if necessary, as determined in these cases. Also, oral appliance therapy, genioglossus muscle advancement, and maxillomandibular advancement may also be conducted. In addition, patients with OSA are medicated and treated with positive airway pressure therapy in the neurology department.

\section{Institution's Protocol for UACT}

All subjects in the study undergo non-contrast CT scanning with a 320-detector-row scanner for an airway evaluation. The UACT obtains axial, coronal, and sagittal images of the entire upper airway, from the base of the skull to the carina level of the distal trachea (Fig. 1). All datasets were processed with iterative reconstruction with a $3 \mathrm{~mm}$ slice thickness and a $3 \mathrm{~mm}$ interval.

\section{RESULTS}

In this relation, it was found that lesions irrelevant to sleep respiratory disturbance were observed in 75 (19.5\%) of the 378 patients studied. Of these patients, 59 were male and 15 were female, and they ranged in age from 14 years to 79 years (average: 47.8 years). Notably, the disease with the highest incidence rate was paranasal sinusitis $(n=32 ; 8.4 \%)$ (Fig. 2$)$, followed by thyroid disease $(n=16 ; 4.2 \%)$, lymph node enlargement $(n=6$; $1.5 \%$ ), brain lesion ( $n=6 ; 1.5 \%$ ) (Fig. 3 ), pulmonary tuberculosis ( $\mathrm{n}=3,0.7 \%)$ (Fig. 4), vallecular cyst $(\mathrm{n}=2 ; 0.5 \%)$, lung nodule $(\mathrm{n}=1 ; 0.2 \%)$, and miscellaneous $(\mathrm{n}=9 ; 2.3 \%)$ (Table 1$)$. Of the 74 patients with sleep apnea who underwent UACT, 25 (6.61\%) required treatment; $14(3.7 \%)$ were given medication, and $11(2.91 \%)$ underwent surgery. Of the 32 patients with pa-
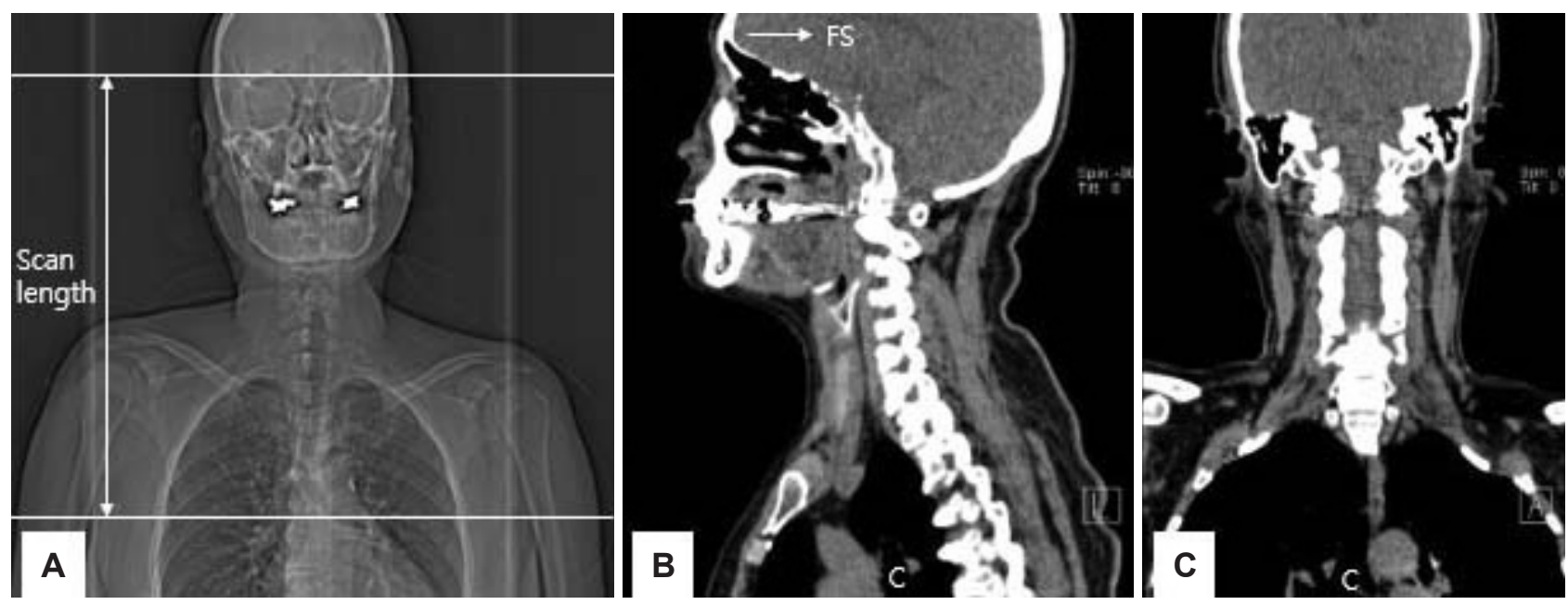

Fig. 1. Representative images of airway CT scans. A: The CT scans start from the FS to the C, including both the upper airway and trachea. B: Sagittal CT images. C: Coronal CT images. FS: frontal sinus of the skull, C: carina level of the distal trachea. 

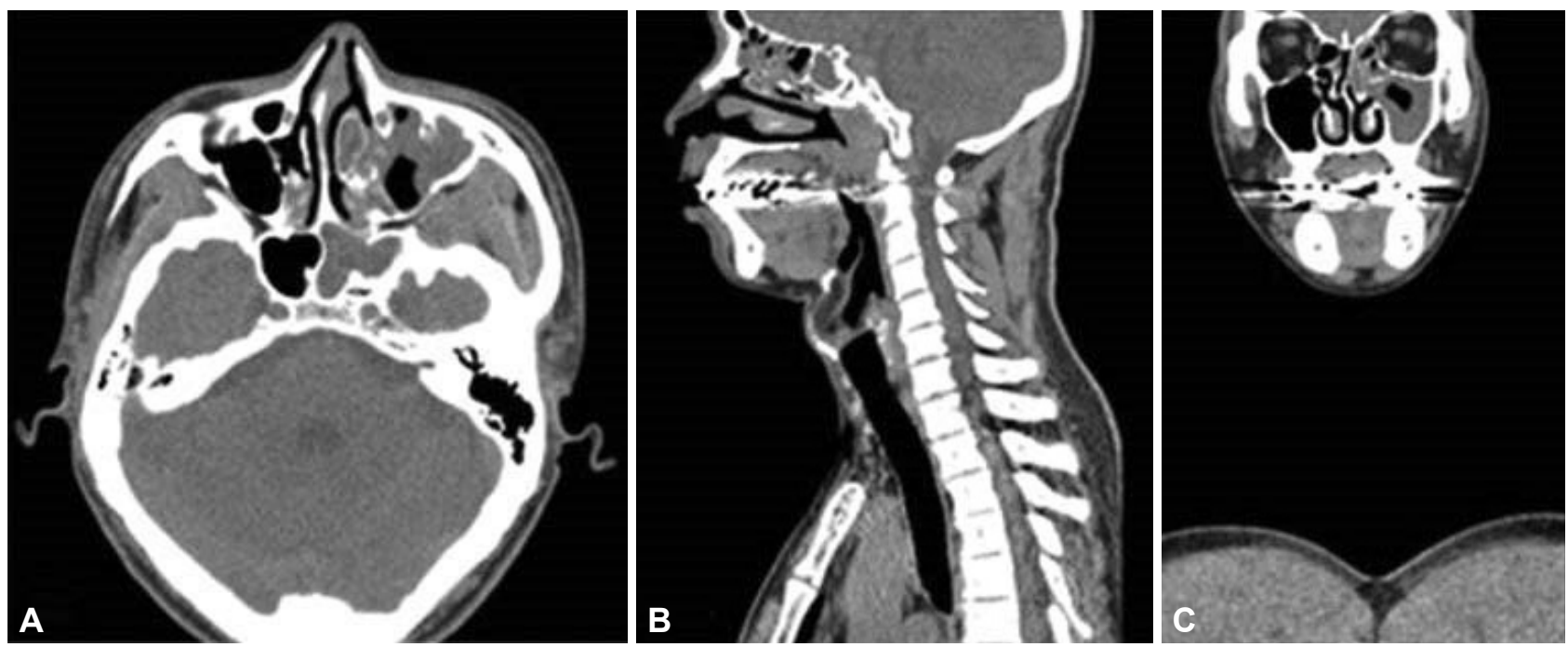

Fig. 2. Paranasal sinusitis. Axial (A), coronal (B), and sagittal (C) CT images of the paranasal sinus showed opacification of left maxillary, ethmoid and sphenoid sinuses.
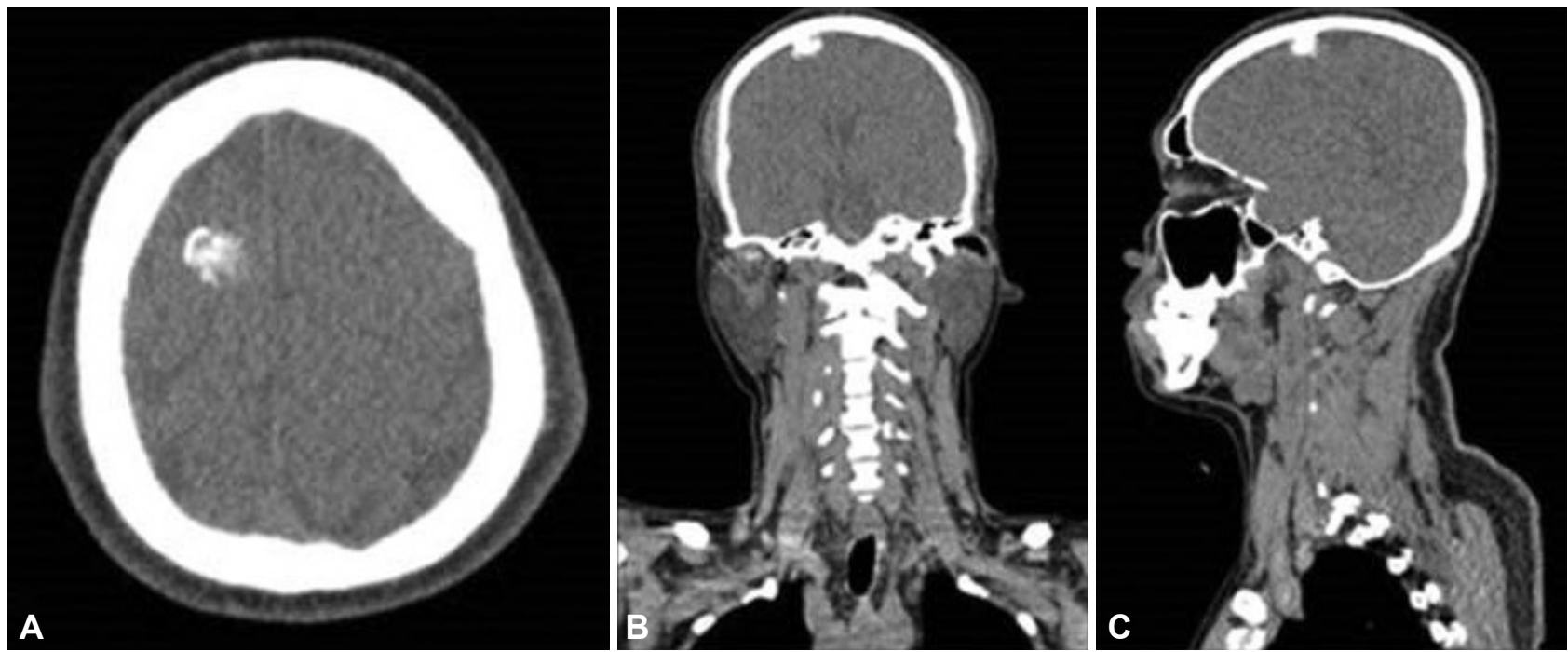

Fig. 3. Calcified meningioma. Axial (A), coronal (B), and sagittal (C) CT images of calcific nodular lesions found in vertex portion adjacent to right frontal lobe.
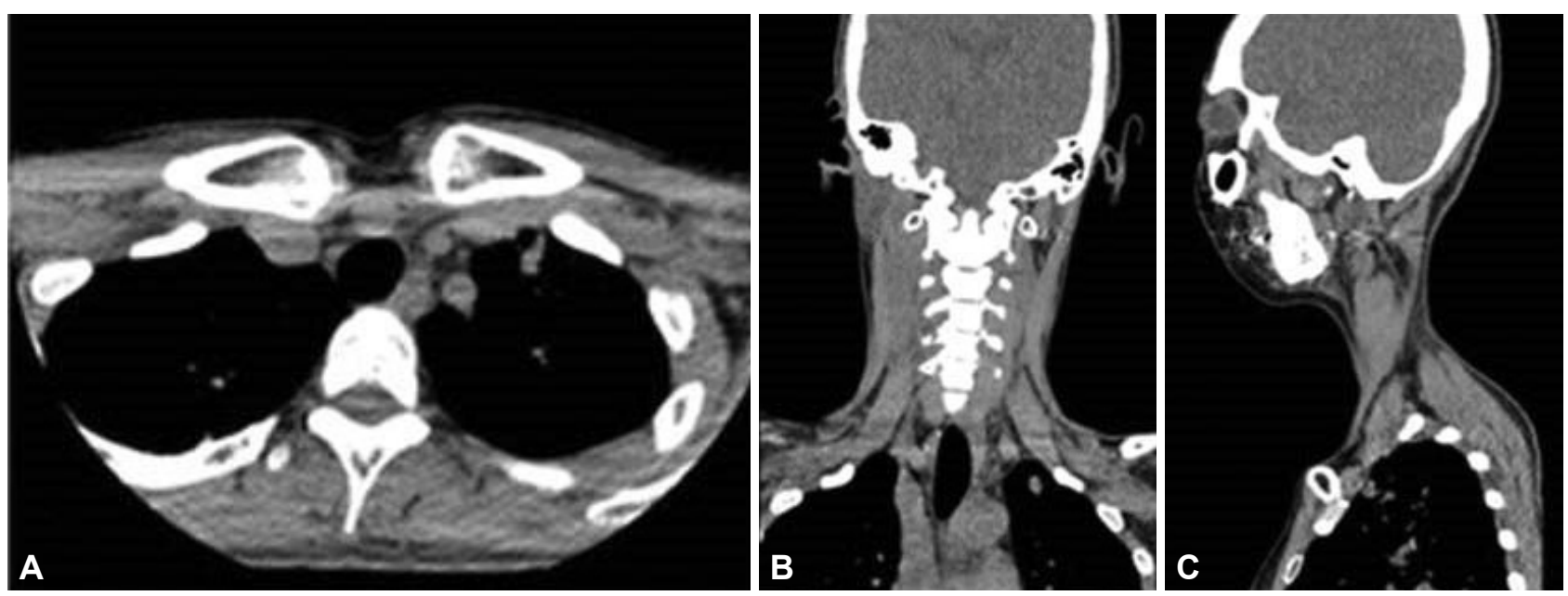

Fig. 4. Active pulmonary tuberculosis. Axial (A), coronal (B), and sagittal (C) CT images of multiple centrilobular nodules in peribronchial distribution with multiple non-calcified nodules and consolidation in both lung. 
ranasal sinus, 13 took medication, 9 underwent non-endoscopic paranasal sinus operation, 7 are being followed up, and 3 were lost during follow-up. Next, of the 16 patients with thyroid disease, 1 was diagnosed with thyroid papillary carcinoma and underwent total thyroidectomy, 13 are being followed up, and 2 were lost during follow-up. Finally, of the two patients with vallecular cyst, one underwent dissection and one patient with pulmonary tuberculosis was given tuberculosis agents for active tuberculosis (Fig. 5).

\section{DISCUSSION}

The fundamental problem is that unanticipated lesions that are located in areas other than those planned for observations are sometimes overlooked. These lesions can contain significant information that can influence treatments. The utilization of CT has led to the discovery of unanticipated lesions; in particular, UACT can show collateral lesions incidentally as it encompasses large areas starting from the basilar to the upper lung. Similarly, of the 378 patients examined in this study, 75 (19.5\%) showed incidental findings upon UACT analysis.

The most frequently observed incidental findings were shown to be paranasal sinusitis, which was found in 32 patients (8.4\%), and $18-51.8 \%$ paranasal sinus lesions were also observed in other previous studies as incidental findings [6,7]. In general, paranasal sinuses are observed in high quality through UACT and disease in this area can exacerbate sleep-disordered breathing. Some studies report that up to $64.7 \%$ patients with paranasal sinusitis showed evidence of sleep-disordered breathing, and it is noted that OSA patients have a higher risk of developing paranasal sinusitis [8]. Therefore, such lesions can also negatively affect the treatment process by affecting the ventilation pressure when using positive airway pressure therapy. Because the patency of the osteomeatal complex makes up much of the patency of the upper airway, careful care is necessary even when there are no lesions observed in this area [9]. In such an environment, nine patients in this study underwent a paranasal sinus endo-

Table 1. Number of incidental findings on upper airway CT

\begin{tabular}{ccccccccc}
\hline Total & Sinusitis & Thyroid ds. & LNE & Brain ds. & Pulmonary tb & Vallecular cyst & Lung nodule & Others \\
\hline 75 & $32(8.4)$ & $16(4.2)$ & $6(1.5)$ & $6(1.5)$ & $3(0.7)$ & $2(0.5)$ & $1(0.2)$ & $9(2.3)$ \\
\hline
\end{tabular}

Data are presented as $\mathrm{n}(\%)$.

ds: disease, tb: tuberculosis, LNE: lymph node enlargement.

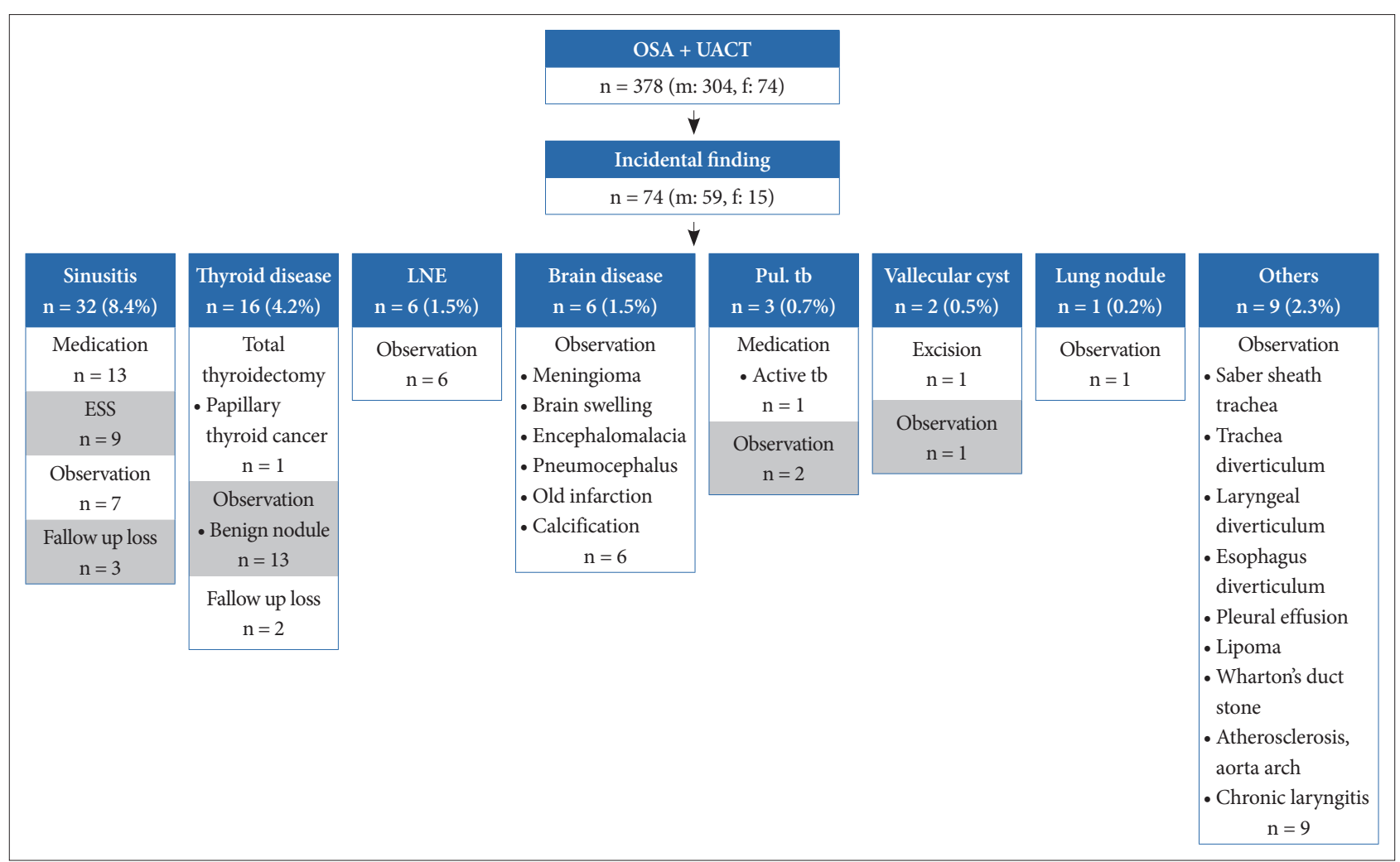

Fig. 5. Summary of incidental findings in UACT. OSA: obstructive sleep apnea, UACT: upper airway CT, LNE: lymph node enlargement, Pul. tb: pulmonary tuberculosis, ESS: endoscopic functional surgery. 
scopic operation, and thirteen were given antibiotic treatment.

Broadly speaking, the thyroid lesions accounted for the second most common incidental findings in this study, and were found in $16(4.2 \%)$ patients. To this end, thyroid incidentaloma has been found in 19-67\% of healthy people with the commercialization of high-resolution ultrasonography [10]. In general, thyroid incidentaloma diagnosed via CT has been reported in $16 \%$ of healthy people, which is higher than the rate of $11.8 \%$ observed in this study [11]. Although more than $90 \%$ of the nodules were benign and did not cause any health problems, 5-10\% are malignant and must be dissected and examined for any signs of a relapse [12,13]. Likewise, one of the sixteen nodules found in this study was malignant and was surgically dissected.

Since UACT images cover the area up to the upper lung, pulmonary tuberculosis prevailing in the upper lung is considered to have a greater chance of being discovered than with the review of the simple nodes. Clearly, in this case its degree of activity was determined in the pulmonary department of the author's institution using high-resolution CT, and was further followed up and treated. Although the vallecular cyst lesions found in two patients were asymptomatic and were not large enough to affect the upper airway patency, one of the two patients requested aggressive care and was further treated by surgical excision. To enhance this understanding, it is noted that the most common brain lesion discovered by chance was a meningioma, and a cerebral basal ganglia calcification was observed in $0.3-0.6 \%$ of the patients in this study [14]. Of the six patients who showed brain lesions, the incidence of a meningioma and basal ganglia calcification were found in one patient respectively. These patients were summarily followed up without any specific treatment noted. Four other patients showed lesions that could be anticipated through their history, and were also followed up without any specific treatment at that time.

Still, it is noted that some institutions use dynamic 3D UACT to localize the upper airway obstruction sites in OSA patients. In particular, it can make a similar effect like 3D image for evaluation of the upper airway. Compared with static UACT which we use in our institution, dynamic 3D UACT provides a highresolution motion of the entire UA simultaneously, which can give crucial information about the dynamic patterns of collapse that are seen in OSA [4]. However, to perform 3D UACT, a higher amount of radiation doses is required for patients, and sometimes a sedation procedure is additionally needed for obtaining accurate images. Fundamentally, our institution performs static UACT together with drug induced sleep endoscopy (DISE). As noted, DISE can be useful in studying the dynamic airway in a sleeping patient with OSA, but there is a limitation in confirming the overall structure of the airway. Since static UACT covers the entire upper airway from the base of the skull to the carina level of the distal trachea, we can check the overall structure of the airway in those cases. Therefore, it is concluded that our institution's UACT can be a cost-effective imaging modality in find- ing obstructive lesions of patients with this condition.

\section{Conclusions}

In particular, of the patients in this study who underwent UACT for sleep respiratory disturbance, $19.5 \%$ showed collateral lesions, $6.61 \%$ required treatment, and $3.7 \%$ were given medication, while $2.91 \%$ underwent surgical treatment. Because UACT provides information in addition to that needed to determine the location of upper airway obstruction, other lesions can be observed, whereby it is likely that some of which will require treatment. Therefore, careful observation and decisionmaking are necessary in these cases.

\section{Acknowledgments}

This work was supported by the Dong-A University research fund.

\section{Conflicts of Interest}

The authors have no financial conflicts of interest.

\section{Authors' Contribution}

Conceptualization: Bae WY. Data curation: Park SH. Formal analysis: Park SH. Investigation: Bae WY. Methodology: Bae WY. Project administration: Bae WY. Supervision: Bae WY. Writing_original draft: Kim S. Writing—review \& editing: Kim S.

\section{REFERENCES}

1. Ryan S, Crinion SJ, McNicholas WT. Obesity and sleep-disordered breathing--when two 'bad guys' meet. QJM 2014;107:949-54.

2. Luo YM, Tang J, Jolley C, Steier J, Zhong NS, Moxham J, et al. Distinguishing obstructive from central sleep apnea events: diaphragm electromyogram and esophageal pressure compared. Chest 2009;135:1133-41.

3. Faber CE, Grymer L. Available techniques for objective assessment of upper airway narrowing in snoring and sleep apnea. Sleep Breath 2003; 7:77-86.

4. Fleck RJ, Ishman SL, Shott SR, Gutmark EJ, McConnell KB, Mahmoud $\mathrm{M}$, et al. Dynamic volume computed tomography imaging of the upper airway in obstructive sleep apnea. J Clin Sleep Med 2017;13:189-96.

5. Alsufyani NA, Flores-Mir C, Major PW. Three-dimensional segmentation of the upper airway using cone beam CT: a systematic review. Dentomaxillofac Radiol 2012;41:276-84.

6. Pette GA, Norkin FJ, Ganeles J, Hardigan P, Lask E, Zfaz S, et al. Incidental findings from a retrospective study of 318 cone beam computed tomography consultation reports. Int J Oral Maxillofac Implants 2012; 27:595-603.

7. Cağlayan F, Tozoğlu U. Incidental findings in the maxillofacial region detected by cone beam CT. Diagn Interv Radiol 2012;18:159-63.

8. Mahdavinia M, Schleimer RP, Keshavarzian A. Sleep disruption in chronic rhinosinusitis. Expert Rev Anti Infect Ther 2017;15:457-65.

9. Nakata S, Noda A, Yasuma F, Morinaga M, Sugiura M, Katayama N, et al. Effects of nasal surgery on sleep quality in obstructive sleep apnea syndrome with nasal obstruction. Am J Rhinol 2008;22:59-63.

10. Tan GH, Gharib H. Thyroid incidentalomas: management approaches to nonpalpable nodules discovered incidentally on thyroid imaging. Ann Intern Med 1997;126:226-31.

11. Jin J, McHenry CR. Thyroid incidentaloma. Best Pract Res Clin Endocrinol Metab 2012;26:83-96.

12. Hegedüs L. Clinical practice. The thyroid nodule. N Engl J Med 2004; 351:1764-71.

13. Yoon DY, Chang SK, Choi CS, Yun EJ, Seo YL, Nam ES, et al. The prevalence and significance of incidental thyroid nodules identified on computed tomography. J Comput Assist Tomogr 2008;32:810-5.

14. Eskandary H, Sabba M, Khajehpour F, Eskandari M. Incidental findings in brain computed tomography scans of 3000 head trauma patients. Surg Neurol 2005;63:550-3. 